\title{
Atypical Trigger Finger: First Manifestation of Gout
}

\author{
David Chen Hol Chieng1* ${ }^{*}$ Xiyuan Ang1, Chee Chien Teoh ${ }^{1}$, Mohamad Hafiz Mohmad Hassim² \\ ${ }^{1}$ Department of Trauma and Orthopaedic, Hospital Bintulu, Bintulu, Malaysia \\ ${ }^{2}$ Orthopaedic Department, International Islamic University Malaysia, Kulliyyah of Medicine, Bandar Indera Mahkota Campus, \\ Kuantan, Malaysia \\ Email: *davidchieng_89@hotmail.com
}

How to cite this paper: Chieng, D.C.H., Ang, X.Y., Teoh, C.C. and Hassim, M.H.M. (2018) Atypical Trigger Finger: First Manifestation of Gout. Open Journal of Orthopedics, 8, 423-428.

https://doi.org/10.4236/ojo.2018.811044

Received: October 17, 2018

Accepted: November 9, 2018

Published: November 12, 2018

Copyright (C) 2018 by authors and Scientific Research Publishing Inc. This work is licensed under the Creative Commons Attribution International License (CC BY 4.0).

http://creativecommons.org/licenses/by/4.0/

\begin{abstract}
Gout is a form of inflammatory arthritis caused by hyperuricemia characterized by painful, hot and swollen joints. However, tophi involving carpal tunnel with concomitant presence of finger movement dysfunction as the first presentation of gout is rare. This suggests the involvement of flexor tendon inside the carpal tunnel. Here, we report a case of infiltration of tophi at the flexor tendon of the hand presented as the first clinical manifestation of gout causing trigger finger like symptoms. Patient underwent surgery to confirm the diagnosis and improve flexor tendon excursion. Our patient's finger movement improved dramatically after operation.
\end{abstract}

\section{Keywords}

Flexor Digitorum Superficialis Tendon, Tophaceous Gout, Trigger Finger, Rare

\section{Introduction}

Gout is one the commonest inflammatory arthropathy, affecting more than 8 million Americans [1]. The disease characterized by painful joint inflammation, frequently involves first metatarsophalangeal joint [2]. Diagnosis is often made clinically and confirmed by identification of monosodium urate crystal in the synovial fluid of the affected joint. Tophi is normally present in long term disease and generally located in elbow, ear pinna and Achilles tendon. Tophi involve the flexor tendons in the hand as the first presentation of gout is uncommon [3]. Rarity of this injury leads to scarcity of reports detailing its management and outcome. Due to its rarity, it is often not included in the differential diagnosis of stenosingtenosynovitis. We report a case of tophaceous gout infil- 
trating flexor tendon at the wrist causing finger movement dysfunction.

\section{Case Report}

42 years old gentleman with no known medical illness was presented to the orthopedic out-patient clinic with complaint of inability to extend his left ring finger which progressively is worsen. There is no tingling sensation or numbness at the left hand. He reported no prior episodes of gouty arthritis and not on regular medication.

Clinically, there was a palpable firm to hard mass over the volar aspect of the left wrist. He had flexion contracture at the proximal interphalangeal (PIP) joint of the left ring finger with limited passive movement (Figure 1). All routine laboratory blood investigations were withinnormal range, including serum uric acid (394 umol/L). Ultrasonography of left wrist showed well defined oval-shaped lesion with perilesional hypoechogenicity seen at the medial aspect of the left wrist. The lesion measuring $0.9 \mathrm{~cm} \times 1.8 \mathrm{~cm} \times 1.9 \mathrm{~cm}$, overlying the bone arising from the flexor tendon. There is no significant finding on the $\mathrm{x}$ ray of the left wrist.

First, we proceeded with A1 pulley release of the left ring finger. However, the finger still remained in flex position. We then proceeded with extended carpal tunnel release. An extended zigzag incision was made from the left palm to the distal forearm to explore the mass proximal to the flexor retinaculum. We identified a chalky white mass infiltrated the flexor digitorum superficialis of the ring finger with hypertrophy of the flexor tendon (Figure 2). The gouty tophus sits mostly on the proximal of the flexor retinaculum, preventing the flexor tendon from gliding through the flexor retinaculum. Flexor tenosynovectomy and excision of the gouty tophus was performed.

Histopathological examination of intra operative specimen confirmed the diagnosis of gouty tophus. Patient was followed up closely in both orthopaedic and internal medicine clinic for 6 months. To reduce the likelihood of recurrence, patient was started on Allopurinol and advised to limit consumption of purine rich food and avoid alcoholic beverages. After aggressive physiotherapy post operatively, the patient recovered and regained full range of motion of his left ring finger (Figure 3 and Figure 4).

\section{Discussion}

Gout is an arthritis cause by excessive uric acid in the blood resulting in deposition of monosodium urate crystal in the joints. Uric acid is a byproduct of purine breakdown. It is excreted by the kidneys. Overproduction of uric acid or reduce excretion of uric acid can lead to gout. The former is the more common cause of hyperuricemia.

Deposition of crystalline uric acid into joints and surrounding tissues leads to formation of tophi. Tophi can erode the bone or cause compression to the surrounding structure. It is commonly found at the external ear, olecranon bursa, 
Achilles tendon and first metatarsophalangeal joint. Rarely, it is found in atypical places such as tarsal tunnel, second metacarpal and wrist flexor tendon [3]. Only around $0.8 \%$ to $2 \%$ of gout first presented with wrist symptoms [4]. Patients who do not receive any treatment for gout will have 19\% - 30\% chance of developing tophi in the wrist [4].

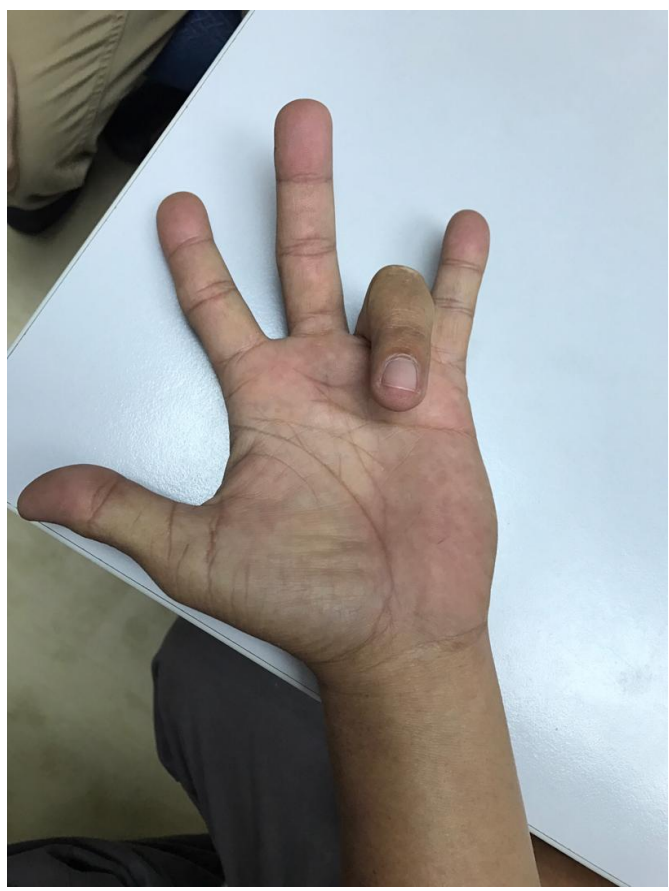

Figure 1. Initial presentation with flexion deformity of the ring finger.

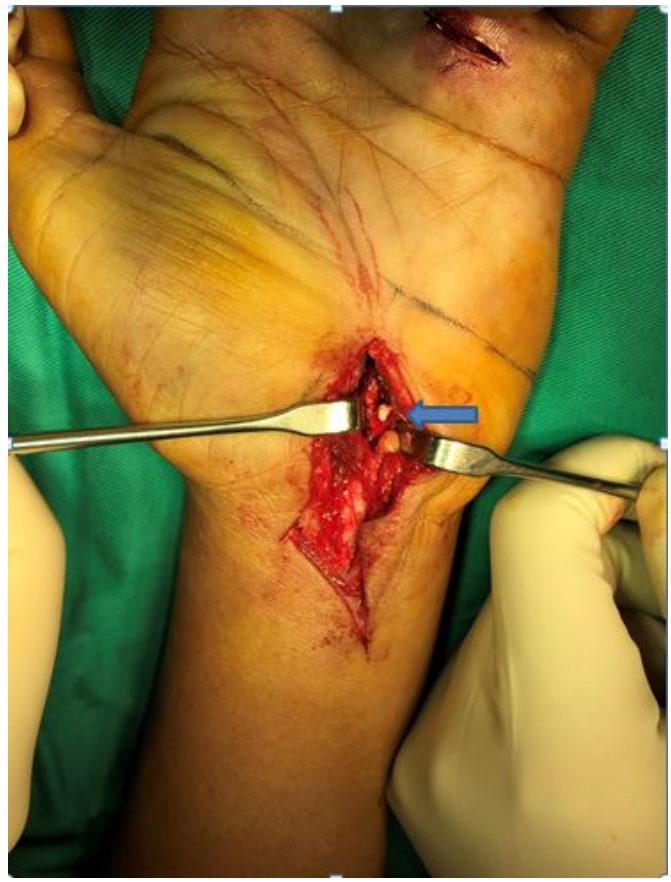

Figure 2. Intraoperative finding of chalky white material in flexor digitorum superficialis tendon sheath. 


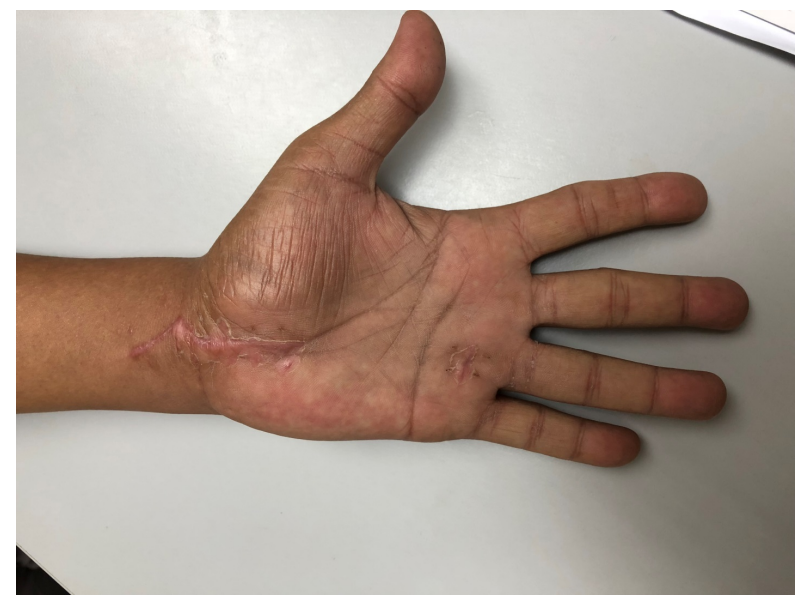

Figure 3. Patient regains full range of motion of left ring finger with ability to fully extend the ring finger.

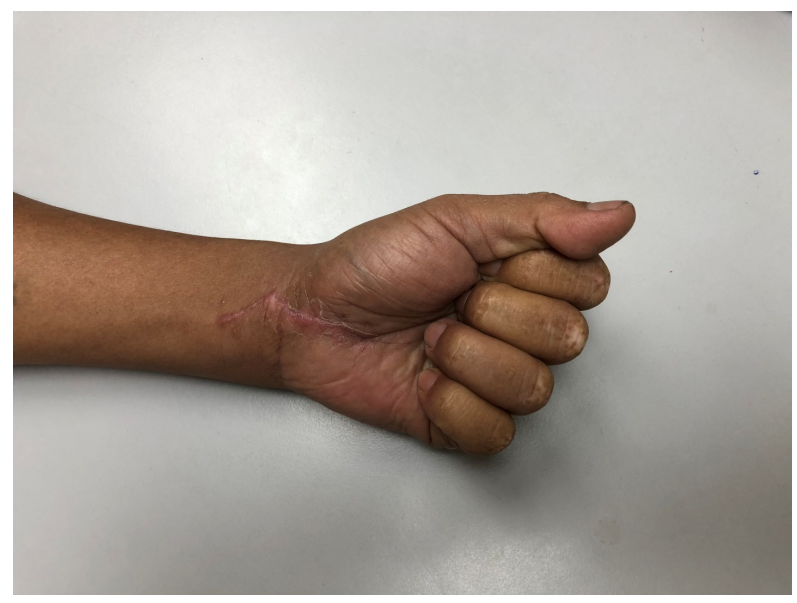

Figure 4. Full recovery of left hand function after operation.

Tophi involving the flexor tendons of the hand as the first manifestation of gout are rare as its development correlate with disease duration [5]. Concomitant presence of finger movement dysfunction suggests involvement of the flexor tendons in the carpal tunnel. Tophi can cause compression to surrounding structure including median nerve due to mass effect. There are only a handful of reports of intratendinous infiltration of tophaceous gout involved predominantly at wrist and co-existed with carpal tunnel syndrome. Kumar et al. and Sano et al. both described an isolated atypical trigger finger due to the flexor tendon tophaceous infiltration proximal to the flexor retinaculum [4] [6]. Similar to their case, our case has tophus at the proximal of the flexor retinaculum. Thus, causing flexor contracture of the ring finger but spared the median nerve. Surgery is recommended to confirm the diagnosis, to decompress the median nerve, and for immediate improvement of flexor tendon excursion [7]. Partial removal of gouty tophus and retaining the integrity of the tendon provide a good outcome in our case. Medical treatment of gout should be commenced as soon as possible once diagnosis established to control serum urate level and prevent further de- 
positions [7] [8]. Allopurinol is started to lower the serum urate and prevent further depositions.

Sano K et al. and Hyun-Sook Kim et al. both reported a case of atypical triggering at the wrist and severe carpal tunnel syndrome caused by tophaceous gout respectively [6] [9]. In their report, patient is known case of gout with present of tophi in other body parts. Hence, the diagnosis is more obvious. Our patient has no previous history of gouty arthritis with normal serum uric acid. Hence, gouty tenosynovitis is at the bottom of our differential diagnosis list. Measurement of serum uric acid level is not the definitive tool in diagnosing gout. Snaith and Coomes et al. found a normal serum uric acid in $17 \%$ of acute episodes of gout [10]. Detailed history, clinical examination, laboratory investigations and imaging can aid in achieving the diagnosis.

\section{Conclusion}

Most of the cases of gout first are presented as painful joints. Tophi mostly present in those longstanding cases and first presentation of it in patients who is not known to have gout is rare. When tophi cause significant morbidity and surgery is required, its aim is mainly to reduce the mass effect to the underlying structure and improve the gliding of the tendon. Delay in treatment may lead to deformity and impair function of the hands. Combination of surgical and medical treatment can lead to improved clinical outcomes.

\section{Conflicts of Interest}

The authors declare no conflicts of interest regarding the publication of this paper.

\section{References}

[1] Zhu, Y., Pandya, B.J. and Choi HK. (2011) Prevalence of Gout and Hyperuricemia in the US General Population: The National Health and Nutrition Examination Survey 2007-2008. Arthritis \& Rheumatism, 63, 3136-3141. https://doi.org/10.1002/art.30520

[2] Pillinger, M.H., Goldfarb, D.S. and Keenan, R.T. (2010) Gout and Its Comoribidities. Bulletin of the NYU Hospital for Joint Diseases, 68, 199-203.

[3] Meyer zu Reckendorf, G. and Lupascu, D. (2010) Flexor Tendons Involvement in Gout. A Case Report and Review of Literature. Chirurgie de la Main, 29, 199-202. https://doi.org/10.1016/j.main.2010.04.004

[4] Kumar, R., Sahni, V.K. and Jauhar, S. (2015) Finger Flexion Contracture: First Manifestation of Gout. Journal of Orthopaedic Case Report, 5, 66-68.

[5] Wernick, R., Winkler, C. and Campbell, S. (1992) Tophi as the Initial Manifestation of Gout. Report of Six Cases and Review of Literature. Archives of Internal Medicine, 152, 873-876. https://doi.org/10.1001/archinte.1992.00400160155032

[6] Sano, K., Kohakura, Y., Kimura, K. and Ozeki, S. (2009) Atypical Triggering at the Wrist Due to Intratendinous Infiltration of Tophaceous Gout. HAND, 4, 78-80. https://doi.org/10.1007/s11552-008-9120-4

[7] Hernandez Cortes, P., Caba, M., Gomez Sanchez, R. and Gomez Morales, M. (2011) 
Digital Flexion Contracture and Severe Carpal Tunnel Syndrome Due to Tophaceous Infiltration of Wrist Flexor Tendon: First Manifestation of Gout. Orthopaedics, 34, e797-e799.

[8] Akizuki, S. and Matsui, T. (1984) Entrapment Neuropathy Caused by Tophaceous Gout. Journal of Hand Surgery (European Volume), 9, 331-332.

https://doi.org/10.1016/0266-7681(84)90054-8

[9] Kim, H.-S. (2014) Carpal Tunnel Syndrome Caused by Tophaceous Gout. The Korean Journal of Internal Medicine, 29, 544-545.

https://doi.org/10.3904/kjim.2014.29.4.544

[10] Snaith, M.L. and Coomes, E.N. (1977) Gout with Normal Serum Urate Concentration. BMJ, 1, 686-687. https://doi.org/10.1136/bmj.1.6062.685-a 